Avrupa Bilim ve Teknoloji Dergisi

Özel Sayı, S. 452-456, Nisan 2020

(C) Telif hakkı EJOSAT'a aittir

Araștırma Makalesi
European Journal of Science and Technology

Special Issue, pp. 452-456, April 2020

Copyright C 2020 EJOSAT

Research Article

\title{
LSTM Mimarisi Kullanarak USD/TRY Fiyat Tahmini***
}

\author{
Özlem Alpay ${ }^{1 *}$ \\ ${ }^{1}$ Gazi Üniversitesi, Mühendislik Fakültesi, Bilgisayar Mühendisliği Bölümü, Ankara, Türkiye (ORCID: 0000-0002-5432-4102)
}

(Konferans Tarihi: 5-7 Mart 2020)

(DOI: 10.31590 /ejosat.araconf59)

ATIF/REFERENCE: Alpay, Ö. (2020). LSTM Mimarisi Kullanarak USD/TRY Fiyat Tahmini. Avrupa Bilim ve Teknoloji Dergisi, (Özel Say1), 452-456.

\section{$\ddot{O} z$}

Son zamanlarda, derin öğrenme yaklaşımlarının hızlı bir şekilde gelişmesi bu konuya olan ilgiyi arttırmış ve birçok alanda başarılı bir şekilde uygulanmaya başlanmıştr. Bu alanlardan birisi de finansal zaman verileridir. Finansal varlıkların fiyatını tahmin etmek, doğru tahminlerle yatırım karar verme riskini azaltabileceğinden önemlidir. LSTM (Uzun kısa süreli bellek), zaman serilerindeki önemli aralık ve uzun gecikme olaylarını işleyip tahmin etmek için uygun ve sıralı verilerde kullanılan yeni bir algoritmadır. Değerlendirmeler 1/1/2000 - 12/31/2017 tarihleri arasında USD/TRY paritesi veri seti kullanılarak gerçekleştirilmiştir. Yapılan çalışmalar sonucunda LSTM yaklaşımının başarılı, gerçek değerlere daha yakın bir tahmin yaptığı görülmüştür. Bunun nedeni LSTM mimarisinin dahili bir belleğe sahip olup girişini hatırlayabilmesidir. Bu makale de LSTM mimarisinin zamansal özelliklere dayanmasından dolayı zamansal verilerin (stok verileri, finansal veriler vb) tahmin sürecinde başarılı bir şekilde uygulanabilir olduğu gözlenmiştir.

Anahtar Kelimeler: Finansal Veriler, Sinir Ağı, LSTM

\section{USD / TRY Price Prediction Using LSTM Architecture}

\begin{abstract}
Recently, the rapid development of deep learning approaches has increased the interest in this subject and has started to be applied successfully in many areas. One of these areas is financial time data. Prediction a financial asset's price is important as one can lower the risk of investment decision- making with accurate prediction. LSTM (Term Memory Long-Short) is suitable for processing and predicting the important events of interval and long delay in time series and a new algorithm used in sequential data. The evaluations was conducted using between 1/1/2000 - 12/31/2017 using USD / TRY parity dataset. As a result of the studies, it was seen that the LSTM approach made successful and closer predict to the real values. This is because the LSTM architecture has an internal memory and can remember its input. In this article, it has been observed that temporal data (stock data, financial data etc.) can be applied successfully in the prediction process since LSTM architecture is based on temporal properties.
\end{abstract}

Keywords: Financial Data, Neural Network, LSTM

\footnotetext{
* Özlem ALPAY: Gazi Üniversitesi,Mühendislik Fakültesi, Bilgisayar Mühendisliği Bölümü, Ankara, Türkiye, ORCID: 0000-0002-5432-4102 ozlemalpay@gazi.edu.tr
}

* Bu makale International Conference on Access to Recent Advances in Engineering and Digitalization (ARACONF 2020) de sunulmuştur. 


\section{Giriş}

Zamana bağlı, zaman sıralı olarak gelen verilerinin analizi, zaman serisi verilerinin analizi olarak adlandırılmaktadır [1]. Son zamanlardaki gelişmeler ile, finansal alanda, borsa ve piyasa işlemlerinde makine öğrenme, yapay zeka uygulamaları kullanılmaya başlanmıştır [2].

Borsa tahmini, döviz kuru, banka iflasları, finansal riski anlama ve yönetme, alım satım vadeli işlemleri, kredi notu, kredi yönetimi, banka müşteri profili oluşturma ve kara para aklama analizleri veri analizi için temel finansal görevlerdir.

Faydalı örüntüler bulma sürecinde, korelasyonların finansal modellemede önemli bir yeri vardır. Doğrusal ve doğrusal olmayan modeller, çok katmanlı sinir ağları, k-means ve hiyerarşik kümeleme, k-en yakın komşu, karar ağacı analizi, regresyon (lojistik regresyon, genel çoklu regresyon), ARIMA, temel bileşen analizi ve Bayes öğrenmesi, ilişkisel yöntemler, olasılık yöntemleri, destek vektör makinesi, gizli Markov modelleri gibi birçok veri analizi yöntemi ve tekniği finansal modellemede kullanılmıştır [3].

$\mathrm{Bu}$ amaçla çeşitli algoritma ve yöntemler geliştirilmiş ve geliştirilmeye devam etmektedir. Son yıllarda derin öğrenme algoritmalarının tahmin ve sınıflandırma gibi çeşitli alanlarda kullanılarak iyi performans verdiği görülmektedir.

Hiransha vd, bir şirketin hisse senedi fiyatını mevcut tarihsel fiyatlara göre tahmin etmek için dört tür derin öğrenme mimarisi kullanılmışlardır. Bunlar; MLP (Multi- Layer Perceptron), RNN (Recurrent Neural Network ), LSTM(Long Short Term Memory), ve CNN (Convolutional Neural Network) .Hindistan'ın Ulusal Menkul Kıymetler Borsası (NSE) ve New York Menkul Kiymetler Borsası (NYSE) olarak iki farklı borsada günlük kapanış fiyatı kullanılmıştır. Sistem, NSE'den tek bir şirketin hisse senedi fiyatı ile eğitilmiştir ve hem NSE hem de NYSE'den beş farklı şirket için test edilmiştir. CNN'nin diğer modellerden daha iyi performans gösterdiği görülmüştür [4].

Fischer ve Krauss yaptıkları çalışmada S\&P 500'in kurucu hisse senetleri için 1992'den 2015'e kadar örneklem dişı yön hareketlerini tahmin etmek için LSTM ağları kullanmışlardır. Genel pazara göre olan performans 1992'den 2009'a kadar çok açık, ancak 2010'dan itibaren, işlem maliyetlerinden sonra sıfıra yaklaşan LSTM kârlılığı ile fazla getiri tahkim edildiği gözlenmiştir. Bu bulgulardan yararlanarak, işlem maliyetlerinden önce yüzde 0.23 veren kurallara dayalı kısa vadeli bir geri dönüş stratejisi resmileştirilmiştir [5].

Cheng vd., hisse senedi fiyat hareketini öngörmek ve alım satım stratejileri yapmak için bir LSTM modeli önermişlerdir. Çalışma iki konuya odaklanmıştır. LSTM modelini kullanarak gelecekteki hisse senedi fiyatı hareketini tahmin etmek için tarihsel hisse senedi verileri ve teknik göstergeleri kullanılmışlardır. Çalışmadaki sonuçlar geri kazanımlara göre hesaplanmıştır. Geçmiş fiyat verileri ve teknik göstergeler ile modelin eğitimi yapılmış daha sonra tahmin sonucunu bir ticaret stratejisine karar vermek için kullanılmıştır [6].

Çalışmanın amacı; finansal zaman serisi verilerinde, orta ve uzun vadede finansal tahmin yapabilen, yüksek oranda kar elde edilmesi sağlayabilen, stabil kararlar alabilen metot ve modeller geliştirmek olarak belirlenmiştir.

\section{Materyal ve Metot}

Derin öğrenme, büyük miktarda etiketsiz/denetlenmemiş veriden öğrenmeyi başarılı bir şekilde gerçekleştirebildiğinden, büyük verilerden anlamlı gösterimler ve örüntüler bulmada sıklıkla kullanılmaktadır. En basit tanımıyla derin öğrenme, makine öğrenmesi yöntemlerinin büyük verilere uygulanması olarak ifade edilmektedir. Bu çalışmada finansal tahmin ve sınıflama problemlerinde derin öğrenme hiyerarşik modellerinin nasıl kullanılabileceği araştırılmıştır. Finansal tahmin sorunları - menkul kıymetleri tasarlama ve fiyatlandırma, portföy oluşturma, risk yönetimi gibi konular - genellikle karmaşık veri etkileşimlerine sahip büyük veri setlerini içerdiğinden tam ekonomik model oluşturmak şu an için zor veya imkansızdır. Bu problemlere derin öğrenme yöntemleri uygulandığında, finanstaki standart metotlardan daha faydalı sonuçlar alınabilir. Özellikle, derin öğrenme, en azından şu an için, mevcut herhangi bir finansal ekonomik teori için görünmez olan veri etkileşimlerini algılayabilir ve bunları etkin bir şekilde kullanabilir.

Derin öğrenme yöntemlerinde tahmin ve sınıflandırma gibi süreçlerde çeşitli mimarilerden yararlanılmaktadır. LSTM, bu mimarilerden biridir.

LSTM, RNN tekrarlayan sinir ağlarının belleğini genişleten bir model veya mimaridir. Tipik olarak, tekrarlayan sinir ağları, mevcut sinir ağında kullanılmak üzere kalıcı önceki bilgileri kullanmaları nedeniyle "kısa süreli hafızaya" sahiptir. Esasen, mevcut görevde önceki bilgiler kullanılır.

LSTM'nin kullanılabileceği çok çeşitli uygulama alanları vardır: el yazısı tanıma, zaman serisi anomali tespiti, konuşma tanıma, öğrenme grameri, müzik bestelemek vb.

Bu çalışma kapsamında derin öğrenme mimarisi (LSTM)da zaman serisi alanında kullanılmıştır.

$\mathrm{Bu}$ çalışmada USD/TRY paritesi veri seti olarak kullanılmıştır. Çalışma da, USD/TRY paritesi "https://tr.investing.com/currencies/usd-try" adresinden ulaşılmıştır. İlk olarak, veriseti için önişleme yapılmıştır. Veri seti, eğitim ve test verisi olarak ikiye ayrılmıştır. Eğitim verisi olarak, 1/1/2000 ile 12/31/2016 tarihleri arası 1/1/2017 ile 12/31/2017 tarihleri arasındaki parite, test verisi olarak kullanılmıştır. Veri setinde, tarih, aç1lış kapanış vb. Değerler ile ve günlük hacim bilgisi kullanılmıştır. Veri setinin bir kısmı Tablo 1'de görülmektedir. Veri setindeki özellikler ve değerleri Tablo 2'de verilmiştir. 
Tablo 1. Veriseti alanları ve örnek değerleri

\begin{tabular}{c|c|c|c|c|c|c|}
\hline \hline & A & B & C & D & E & \multicolumn{1}{c|}{ F } \\
\hline 1 & Date & Open & High & Low & Close & Volume \\
\hline 2 & $30 / 12 / 2016$ & 3.5277 & 3.5224 & 3.5325 & 3.5095 & 0.13 \\
\hline 3 & $29 / 12 / 2016$ & 3523 & 3.5395 & 3.5441 & 3.5145 & -0.48 \\
\hline 4 & $28 / 12 / 2016$ & 3.54 & 3522 & 3.5505 & 3517 & 0.53 \\
\hline 5 & $27 / 12 / 2016$ & 3.5214 & 3.5123 & 3.5273 & 3501 & 0.27 \\
\hline 6 & $26 / 12 / 2016$ & 3.5118 & 3518 & 3.5209 & 3.5046 & -0.15 \\
\hline 7 & $23 / 12 / 2016$ & 3.5169 & 3.5121 & 3.5189 & 3.4975 & 0.16 \\
\hline 8 & $22 / 12 / 2016$ & 3.5114 & 3.5091 & 3.5191 & 3.4981 & 0.07 \\
\hline 9 & $21 / 12 / 2016$ & 3.5089 & 3527 & 3.5341 & 3504 & -0.53 \\
\hline 10 & $20 / 12 / 2016$ & 3.5275 & 3.5338 & 3.5425 & 3.5005 & -0.16 \\
\hline
\end{tabular}

Tablo 2. Verisetindeki özellikler ve değerleri

\begin{tabular}{|l|l|}
\hline Özellik & Değerler \\
\hline Date (Tarih) & Date/Month/Year \\
\hline Open (Açılış) & Açılış fiyatı \\
\hline High (Yüksek) & Günün en yüksek değeri \\
\hline Low (Düşük) & Günün en düşük değeri \\
\hline Close & Kapanış fiyatı \\
\hline Volume (Hacim) & Günlük fark \\
\hline
\end{tabular}

\section{Araştırma Sonuçları ve Tartışma}

Önerilen çalışmada, LSTM kullanılarak finansal zaman serisi verilerinin tahmini yapılmaktadır. Literatürde, zaman serisi verilerinin analizinde LSTM kullanımı mevcuttur.

Bu çalışmada elde edilen deneysel sonuçlar için TensorFlow Kütüphanesi kullanılmıştır. TensorFlow, veri akışı grafiklerini kullanarak sayısal hesaplama için açık kaynaklı bir yazılım kütüphanesidir.

LSTM yapısı 4 gizli katmandan ve her gizli katmanda 50 nöron ve çıkış katmanında tek bir çıkış, dropout 0.2 olacak şekilde düzenlenmiştir. Oluşturulan LSTM mimarisi Şekil 1'de gösterilmiştir.

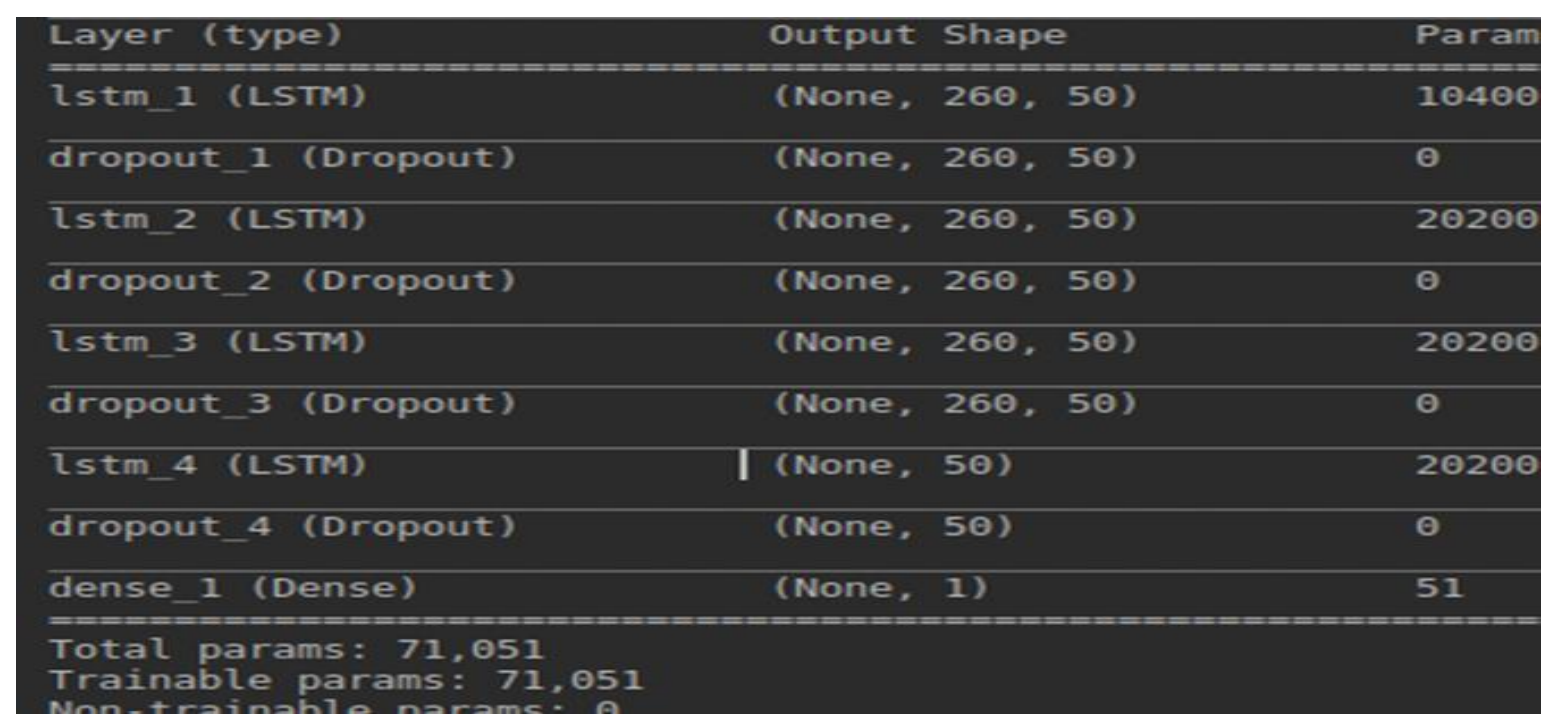

Şekil 1. Oluşturulan LSTM yapısı 
Uygulama 1,10 ve 100 epoch değerlerinde 256 ve 512 batch size değerleri kullanılarak eğitilmiştir. Bu parametre değerlerine göre modelin test verisi ve gerçek veri üzerindeki sonuçları Şekil 2, 3 ve 4'de gösterilmiştir.

Bu çalışmada, USD/TRY fiyat oranlar 3 farklı epoch ve 2 farklı batrch boyutunda tekrarlanmıştır. Şekil 2, Şekil 3 ve Şekil 4'de yapılan tahmin eğrisi ile gerçek fiyat eğrisi karşılaştırılmıştır. Derin öğrenmede mimarilerinde, modellerin başarısını ölçmek için belirli parametreler kullanılmaktadır. Bunlar veri seti boyutu, batch boyutu, epoch sayısı gibi parametrelerdir. Veri setinin parçalar halinde işlenmesi mini-batch olarak tanımlanmaktadır. Batch parametresine verilecek olan değer, modelin aynı anda kaç veriyi işleyebileceğini göstermektedir.

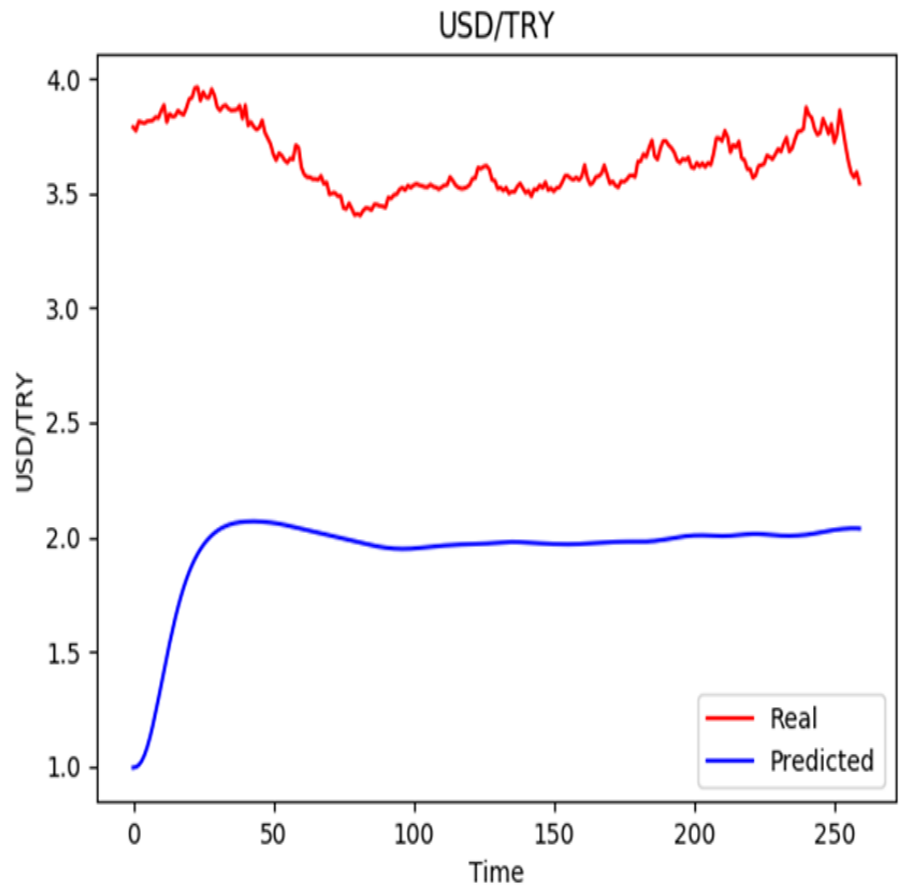

(a)

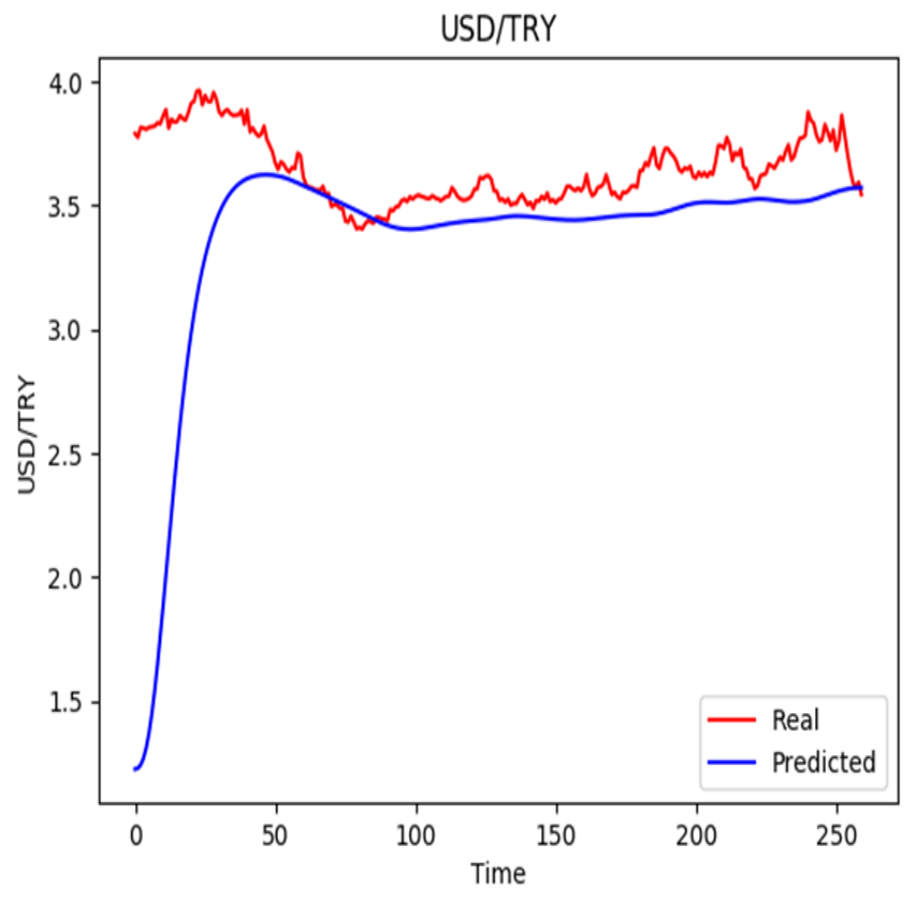

(b)

Şekil 2. Epoch değeri 1 a) batch size 256 b) batch size 512

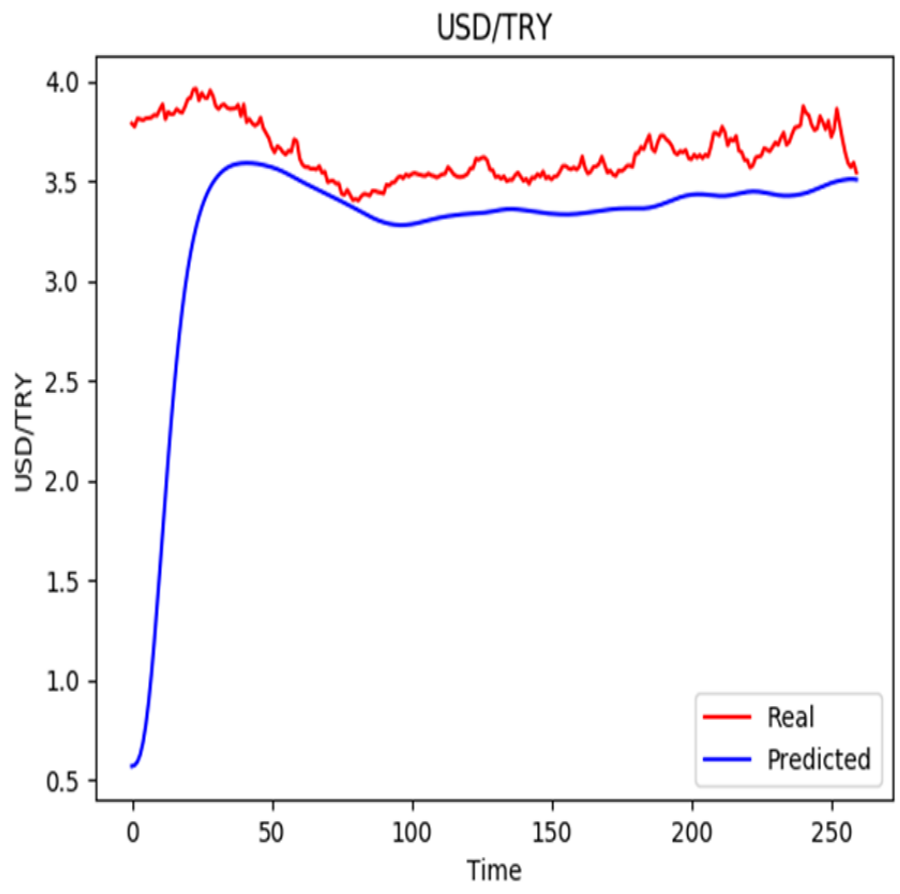

(a)

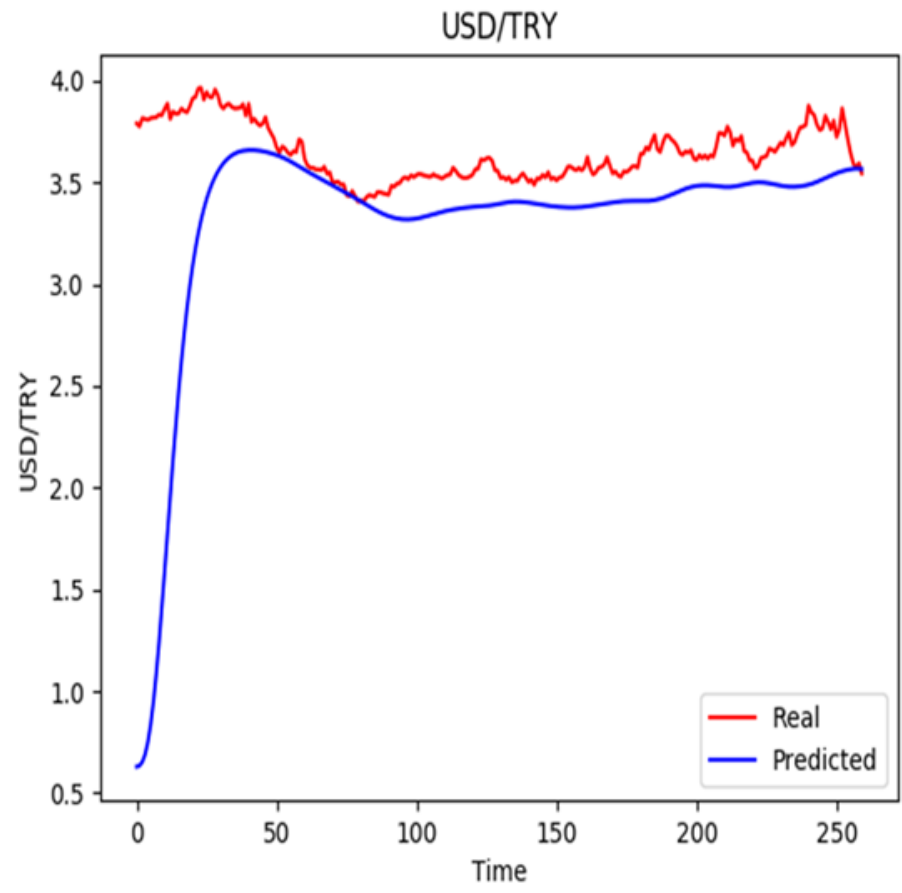

(b)

Şekil 3. Epoch değeri 10 a) batch size 256 b) batch size 512 


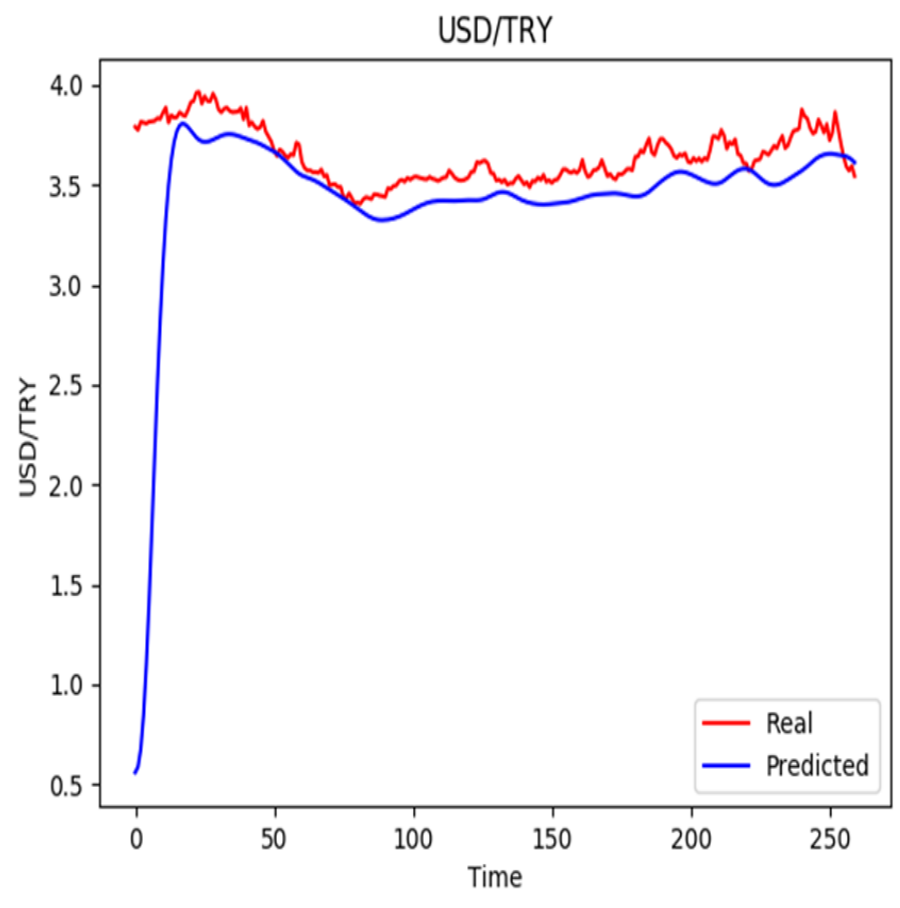

(a)

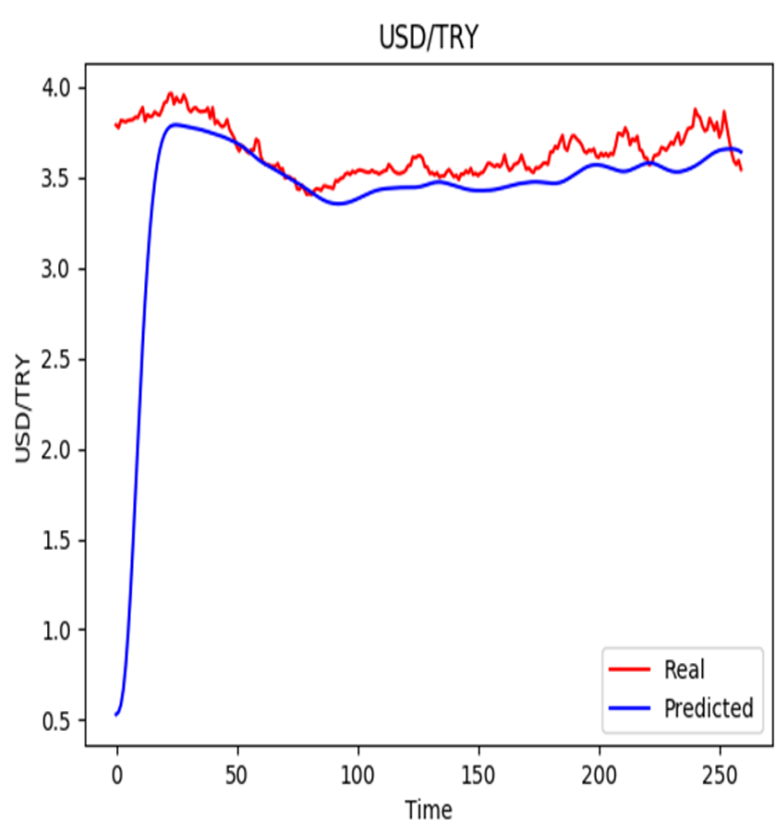

(b)

Şekil 4. Epoch değeri 100 a) batch size 256 b) batch size 512

\section{Sonuç}

$\mathrm{Bu}$ çalışma ile derin öğrenme yaklaşımlarından LSTM mimarisi kullanılarak finans sektöründe, risk ve zarar durumlarının tahminine yönelik bir model geliştirilmiştir. Bu modelde, probleme göre özelleştirilmiş LSTM kullanılarak geçmiş zaman dilimlerindeki değerler kullanılarak belirli bir döneme ait parite değeri tahmin edilmiştir.

Deneysel sonuçlara göre, epoch değerinin artması ile modelin başarısı da artmaktadır. Modelin Başarı oaranı belli bir epoch değerinden sonra çok küçük birimlerde artacağı için bu noktalarda eğitim sonlandırılabilir. Batch değeri küçük seçildiği zaman model kendini ezberleyebilmektedir.Uygun batch size değeri seçildiğinde model gürültüyü daha az öğrenip, kendini ezberlemeyecektir.

Bu süreçte asıl amaç geçmiş verilerin eğiliminden ders alarak ve bu model üzerinde tahmin ederek ertesi günkü finansal değeri modelleyen en iyi konfigürasyon modelini bulmaya çalışmaktadır. Sonuç olarak, belirli epoch ve batch size değerlerinde oluşturulan modelin başarılı bir şekilde fiyat tahmini yaptığı gözlenmiştir.

Çalışma sonucunda şu gibi sonuçlar ortaya çıkmıştır. USD/TRY paritesi ekonomik ve siyasi aksiyonlardan kolay etkilenebilir bir yapıda olduğu için grafik eğitim veri setine göre test işlemi yaptığı için grafik üzerinde dalgalanmalar yaşanmıştır. 2000’li yıllarda USD/TRY paritesi ortalama 0,6 oranından 2016 yılında ise ortalama 3,5 oranına yükselmiştir. Tahmin ettiğimiz 2017 yılında ise ortalama 3,8 oranındadır

Gelecek çalışmalar için endeks olarak birbirine benzeyen paralel değişim (genellikle aynı zaman ve olay çerçevesinde artış veya azalış) gösteren pariteler arasında çalışma yapmaları veya geçmiş verilerin yanında çalışmanın yapıldığı zaman diliminde gerçekleşen siyasi, ekonomik, küresel vb. etkileri de dikkate alan yaklaşımları kullanan çalışma yapmaları önerilir.

\section{Kaynakça}

[1] William W. S. Wei, (2006). Time Series Analysis: Univariate and Multivariate Methods, Pearson Addison Wesley

[2] Cavalcante, R.C., Brasileiroi, R C., Souza, V.L, Nobrega, J.P., Oliveira, A.L.,(2016).Computational intelligence and financial markets: a survey and future directions, Expert Systems With Applications,55,194-211.

[3] Maimonand, O., Rokach, L.,(2005). Data Mining An dKnowledge Discovery Handbook. New York, NY, USA: Springer,doi: 10.1007/b107408.

[4] Hiransha, M., Gopalakrishnan, E.A., Vijay Krishna Menonab, K.P. Soman, (2018). NSE stock market prediction using deeplearning models, Procedia Computer Science, 132,1351-1362

[5] Fischer, T., Krauss, C., (2018). Deep learning with long short-term memory networks for financial market predictions, European Journal of Operational Research, 270, 654-669.

[6] Cheng, L.C., Huang, Y.H., Wu, M.E., (2018). Applied attention-based LSTM neural networks in stock prediction, IEEE International Conference on Big Data (Big Data), Seattle, WA, USA 